Electron beam induced current and scanning tunnelling spectroscopy correlative study of $\mathrm{Cd}_{x} \mathrm{Hg}_{1-x} \mathrm{Te}$ and CdTe crystals

This content has been downloaded from IOPscience. Please scroll down to see the full text. 1998 Semicond. Sci. Technol. 13576

(http://iopscience.iop.org/0268-1242/13/6/007)

View the table of contents for this issue, or go to the journal homepage for more

Download details:

IP Address: 147.96.14.16

This content was downloaded on 14/10/2013 at 15:34

Please note that terms and conditions apply. 


\title{
Electron beam induced current and scanning tunnelling spectroscopy correlative study of $\mathrm{Cd}_{x} \mathrm{Hg}_{1-x} \mathrm{Te}$ and CdTe crystals
}

\author{
G N Panin $\dagger$, C Díaz-Guerra and J Piqueras
}

Departamento de Física de Materiales, Facultad de Físicas, Universidad Complutense, E-28040 Madrid, Spain

Received 13 October 1997, accepted for publication 6 February 1998

\begin{abstract}
A combined scanning electron microscope-scanning tunnelling microscope (SEM-STM) system has been used to characterize $\mathrm{Cd}_{x} \mathrm{Hg}_{1-x} \mathrm{Te}$ and CdTe crystals. The electron beam induced current (EBIC) mode of the SEM shows the existence of inhomogeneities in the electronic behaviour of the samples, mainly related to the presence of subgrain boundaries and precipitates. Current imaging tunnelling spectroscopy images and the related normalized differential conductance curves, obtained with the STM, reveal the electronic inhomogeneities at a finer scale. In particular, local variations of the band gap were shown by the conductance curves in regions with strong EBIC contrast. SEM- and STM-based techniques in a combined instrument appear to be complementary characterization techniques.
\end{abstract}

\section{Introduction}

Cadmium-based binary and ternary II-VI compound semiconductors are attracting interest for their applications as wide-range infrared detectors, solar cells, highperformance photodiodes, injection lasers and other optoelectronic devices. Electrically active impurities and native defects controlling optical and electrical properties of these materials have been investigated in recent years by different spectroscopic and microscopic techniques. In particular, cathodoluminescence (CL) and electron beam induced current (EBIC) in the scanning electron microscope (SEM) have provided space-resolved information on electrically active defects in $\mathrm{CdTe}$ and $\mathrm{Cd}_{x} \mathrm{Hg}_{1-x} \mathrm{Te}$ (e.g. [1-4]).

Scanning tunnelling microscopy (STM) and spectroscopies allow simultaneous investigation of both the structural and the electronic properties of semiconductors with atomic or nanometre resolution in real space. Because of this capability, these techniques are of increasing interest in the characterization of these mentioned materials. No STM studies on $\mathrm{Cd}_{x} \mathrm{Hg}_{1-x} \mathrm{Te}$ have been reported as far as we know, while previous STM work on CdTe refers mainly to surface structure observations. Seehofer et al [5] reported the first atomic resolution images obtained from CdTe (001) samples and described [6] atomic motion on

$\dagger$ Permanent address: Institute of Microelectronics Technology, Russian Academy of Sciences, 142432 Chernogolovka, Moscow District, Russia. that surface. Castro-Rodríguez et al [7] used SEM and STM to study growth patterns in heavily doped CdTe films. The STM measurements of [5] and [6] were performed in ultrahigh vacuum (UHV) while the conditions of [7] were not reported.

In this work the capability of STM and current-imaging tunnelling spectroscopy (CITS) for studying local electronic properties of undoped CdTe crystals, as well as p- and n-type $\mathrm{Cd}_{x} \mathrm{Hg}_{1-x} \mathrm{Te}$ crystals, is investigated. CITS is one of the spectroscopic modes of STM [8] developed by Hamers et al [9] which allows real space imaging of surface electronic states by recording $I-V$ curves at fixed tip-sample separation at every pixel within an image. In addition to the $I-V$ curves themselves, current images can be formed by plotting the measured current at any voltage. Since CITS images are related to the surface electronic structure of the sample, contrast between a metallic and a semiconducting region is expected. This effect has been observed at UHV [10] in CITS images of Fe clusters on GaAs and at high vacuum [11] in the case of metallic deposits on silicon. In [11] the CITS measurements were performed, as in the present work, in a combined STM-SEM system. CITS measurements have also been reported at atmospheric pressure in studies of semiconducting materials (e.g. [12]). Our STM was implemented in an SEM with an EBIC detection system. This experimental arrangement enabled us to study the same area of the sample, visualized through the secondary electron image of the SEM, by both techniques, SEM-EBIC 
and STM. The EBIC images revealed the existence of inhomogeneities in the electronic properties of the samples which are also detected in the same region, at a finer scale, by the tunnelling techniques.

\section{Experimental method}

The samples used were undoped n-type $\mathrm{Cd}_{0.42} \mathrm{Hg}_{0.58} \mathrm{Te}$ $\left(n=2 \times 10^{16} \mathrm{~cm}^{-3}\right)$ and p-type $\mathrm{Cd}_{0.56} \mathrm{Hg}_{0.44} \mathrm{Te}(p=$ $3 \times 10^{15} \mathrm{~cm}^{-3}$ ) crystals, grown by the solid-state recrystallization (SSR) method, and undoped p-type CdTe $\left(p=5 \times 10^{15} \mathrm{~cm}^{-3}\right.$ ) crystals grown by the Bridgman method. For SEM and STM measurements the crystals were cleaved in air and inmediately mounted in the microscope chamber with the surface conveniently oriented to the incidence direction of the SEM electron beam or to the STM tip. The microscope used was a combined SEM-STM instrument, based on a Leica Stereoscan 440 SEM, operating under a vacuum of $1 \times 10^{-6}$ Torr. The small size of the STM allowed us to attach it to the SEM specimen holder. The main features and capabilities of this system are similar to the one previously described in [13]. The cleaved surfaces were observed in the secondary electron (SE) and EBIC modes of the SEM. For EBIC measurements the formation of a barrier, such as a Schottky barrier or a $\mathrm{p}-\mathrm{n}$ junction, is necessary. The EBIC signal is formed by the carriers of different charge separated by the electrical field in the barrier and collected by the contacts. Electron-hole pairs contributing to this signal are only a fraction of those generated by the electron beam of the SEM, as some of the pairs recombine through defect centres before they are able to reach the contacts. Bright contrast in the EBIC images is associated with efficient carrier collection, while dark contrast is due to an enhanced carrier recombination at the defects. After chemical etching of the samples in $2 \%$ bromine methanol, Schottky barriers for EBIC measurements were fabricated by evaporation of $\mathrm{Al}$ on the surface of the crystals. The measurements can be performed in the region covered by the barrier or in its proximity. In the latter case the uncovered surface can be also investigated by STM. Alternatively, when, as in this work, an uncoated untreated surface is investigated, the use of a contact geometry with two ohmic contacts at far opposite ends of the sample appears more appropriate. We also used in the present work this technique, called remote EBIC (REBIC) [14], which has previously been applied to the characterization of II-VI compounds, e.g. [1]. Ohmic contacts, separated by about $2 \mathrm{~mm}$ in the surface, were provided by Au chemical deposition. REBIC signal formation can be described as follows. Electron-hole pairs created by the SEM electron beam are separated by builtin electric fields associated with different charged defects and produce a current in an external circuit. This current is used, after amplification, to image the spatial distribution of electrically active regions in the samples. Detection of the EBIC and REBIC signal was carried out at room temperature with a Matelect ISM-5 system. The energy and current of the SEM electron beam were $17 \mathrm{keV}$ and $2 \mathrm{nA}$ respectively.
Electrochemically etched Pt-Ir tips as well as mechanically cut $\mathrm{Pt}-\mathrm{Ir}$ and $\mathrm{Au}$ tips were used in STM measurements. STM images were recorded in the constantcurrent mode. For CITS measurements the topographic height was recorded at a point of a $128 \times 128$ pixel grid, the feedback loop was interrupted for $2 \mathrm{~ms}$ and the voltage was digitally ramped from the tunnel voltage to a set of 44 predetermined values while the current was sampled. The tip was then moved to the next point and the process repeated. This provides a set of 44 tunnelling current files at different voltages, in addition to the topographic image. The data obtained enable us to plot $I-V$ curves at different points of the image. The tip density of states (DOS) and the tunnelling transmission probability influence the observed dependence of the tunnelling current on tip-sample voltage. The tunnelling current depends exponentially on both tip-sample separation and applied bias voltage. As discussed by Stroscio et al [15], most of this dependence can be removed by computing the ratio of differential to total conductance, $(\mathrm{d} I / \mathrm{d} V) /(I / V)$, which has been claimed to provide a rather direct measure of the surface DOS $[16,17]$. For this reason the $(\mathrm{d} I / \mathrm{d} V) /(I / V)$ curves of the different samples or of different regions of a given sample were recorded.

\section{Results and discussion}

STM images have been obtained from both $\mathrm{Cd}_{x} \mathrm{Hg}_{1-x} \mathrm{Te}$ samples. The images have been found to be reproducible under different working conditions and when the abovementioned different tips were used. In order to perform a correlative study of the topography of our samples, regions with well-defined topographic features were investigated by SEM at high magnification and by STM. An example of these correlative observations is shown in figure 1, which corresponds to a region of a cleaved $\mathrm{Cd}_{0.56} \mathrm{Hg}_{0.44} \mathrm{Te}$ sample containing a set of closely spaced cleavage steps appearing as parallel ridges. The figure shows a series of SEM and STM images of the steps at increasing magnification. The results demonstrate the ability of STM to image $\mathrm{Cd}_{x} \mathrm{Hg}_{1-x}$ Te surfaces under HV conditions. Figure 1(e) represents a composition of the topography image shown in figure $1(c)$ and the corresponding derivative image, revealing grain structures that cannot be clearly appreciated in the topograph owing to the steep background. Measurements performed on the topography image show that the grains have sizes of tens of $\mathrm{nm}$ and heights of about 1-2 nm. To our knowledge there are no previous reports of tunnelling microscopy of this, or a related, ternary II-VI compound, although an atomic force microscopy study was recently carried out on epitaxial $\mathrm{Cd}_{x} \mathrm{Hg}_{1-x} \mathrm{Te}$ [18]. In spite of the low carrier concentration STM images could also be recorded from the CdTe samples, in which steps of about 5-10 $\mathrm{nm}$ height were observed (figure 2).

REBIC images show the existence of inhomogeneities of the electrical properties of the samples. In the REBIC image (figure 3) of the same area shown at higher magnification in figure 1(a), bright-dark stripes are related to the spatially varying surface electrostatic potential. It has previously been reported that the REBIC 

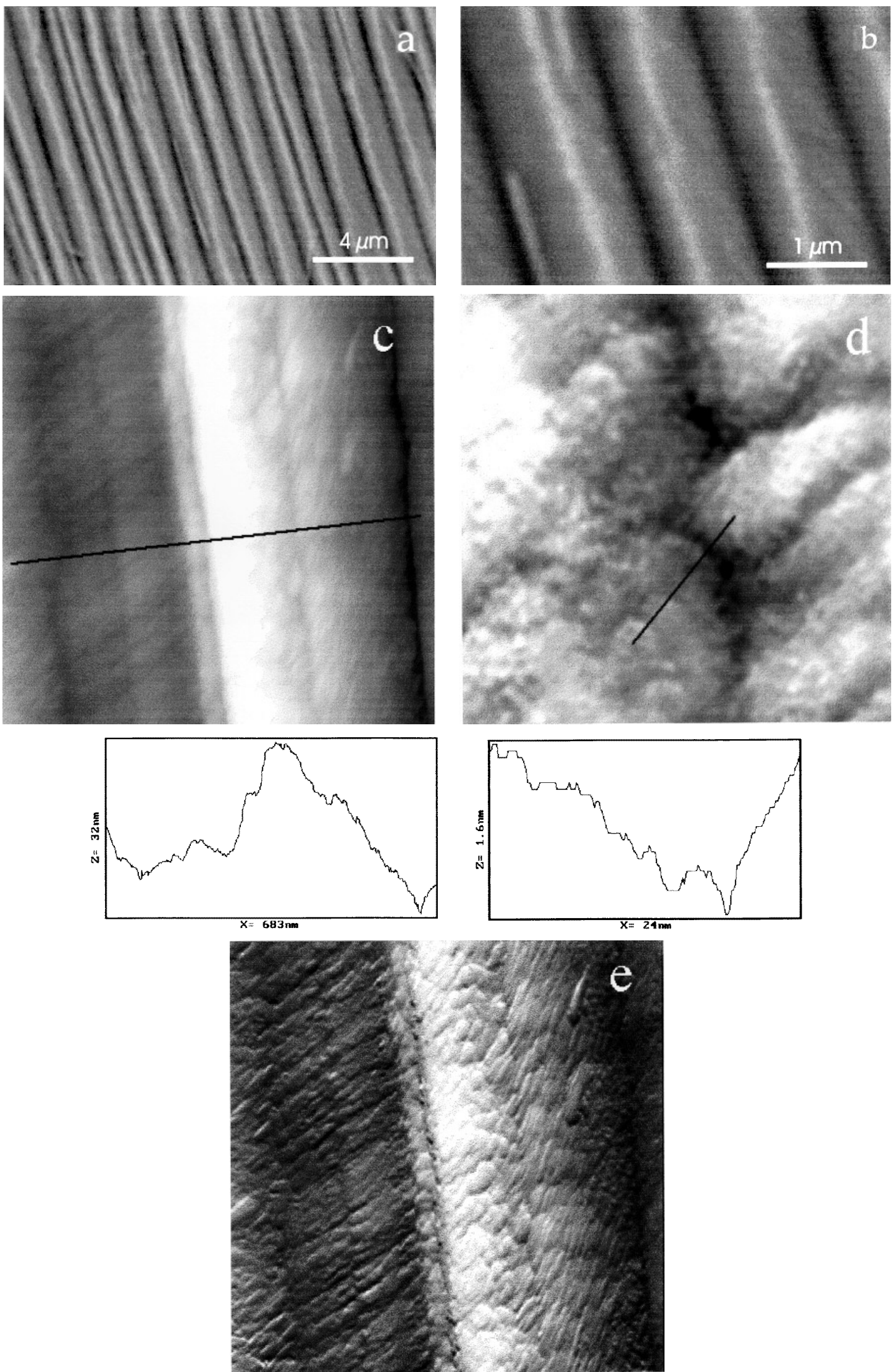

Figure 1. Cleaved surface of a p-type $\mathrm{Cd}_{0.56} \mathrm{Hg}_{0.44}$ Te sample imaged by (a), (b) SEM and (c)-(e) STM. (c) $710 \times 710 \mathrm{~nm}^{2}$, (d) $65 \times 65 \mathrm{~nm}^{2}$; $-1.2 \mathrm{~V}$ sample bias and $0.2 \mathrm{nA}$ tunnelling current for both of these STM images. (e) Image obtained by adding the STM topograph shown in $(c)$ and its derivative. Black lines on the STM images indicate the positions where the height profiles were measured. 

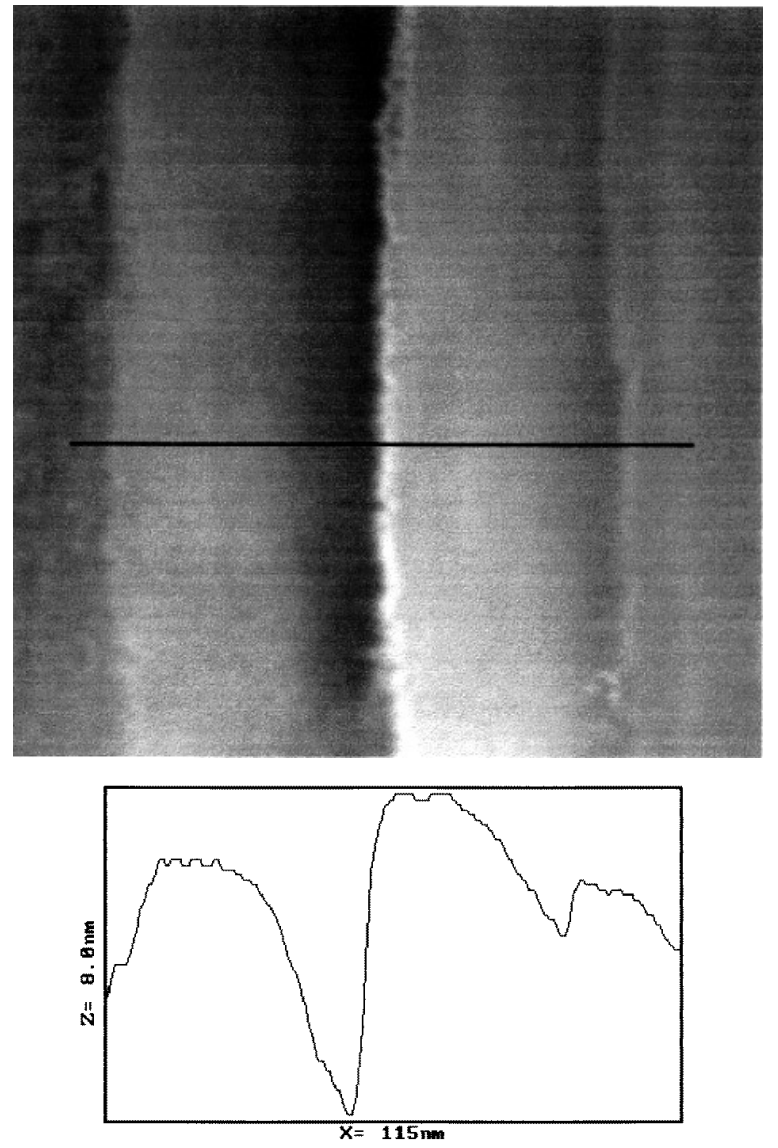

Figure 2. $140 \times 140 \mathrm{~nm}^{2}$ STM image of a CdTe crystal, acquired with a sample voltage of $-1.7 \mathrm{~V}$ and $0.3 \mathrm{nA}$ tunnelling current. The black line on the image marks the position where the height profile was measured.

technique is very useful for detecting potential relief in $\mathrm{PbTe}: \operatorname{In}(\mathrm{Cd})$ heterophase systems [19] and for revealing charged defects in CdTe and $\mathrm{Cd}_{x} \mathrm{Hg}_{1-x}$ Te crystals [20-22]. The bright-dark contrast of the defects shown in figure 3 is due to their built-in potential barriers, which are stable under the electron beam excitation used. The REBIC contrast decreases as the incident beam current increases, indicating a potential barrier reduction with an increase of the excitation level $[20,21]$. EBIC contrast is also observed in CdTe as illustrated in figure 4, which shows a twin boundary that appears as a dark line crossing from top to bottom of the micrograph. In the same figure a distribution of precipitates (dark spots of different sizes) as well as a net of subgrain boundaries are observed. EBIC contrast in these micrographs arises from enhanced carrier recombination at dislocations, grain boundaries and precipitates. The defects were identified by $\mathrm{x}$-ray microanalysis or by analysing the excitation-temperaturedependent EBIC contrast, which is useful to characterize submicron electrical inhomogeneities and structural defects $[20,21]$. The results show that the spot-like defects shown in figure 4 are tellurium precipitates. In addition, the inhomogeneity of the electronic behaviour of the crystals can be observed in the CITS images acquired in the regions where EBIC or REBIC contrast was found. Figure 5 shows a constant-current and three selected CITS images of one, p-type, $\mathrm{Cd}_{0.56} \mathrm{Hg}_{0.44}$ Te sample in which contrast due to local conductance variations is observed. This contrast appears for voltage values below and above the band gap of the sample $(0.64 \mathrm{eV})$ and is higher in the images obtained with positive voltages above the gap value. The observed CITS contrast shows that the inhomogeneity of electronic properties revealed by REBIC is also present at a fine scale which cannot be detected by SEM-based techniques. A similar qualitative appearance of the CITS contrast shown in figure 5 for $\mathrm{Cd}_{x} \mathrm{Hg}_{1-x}$ Te is observed in CITS images of the CdTe samples.

Further information on the sample inhomogeneity is obtained from the normalized differential conductance curves. Curves were plotted from the data of the 44 CITS images by selecting small regions, on which the data were averaged. The main features of the $(\mathrm{d} I / \mathrm{d} V) /(I / V)$ curves appeared also in those obtained from the static

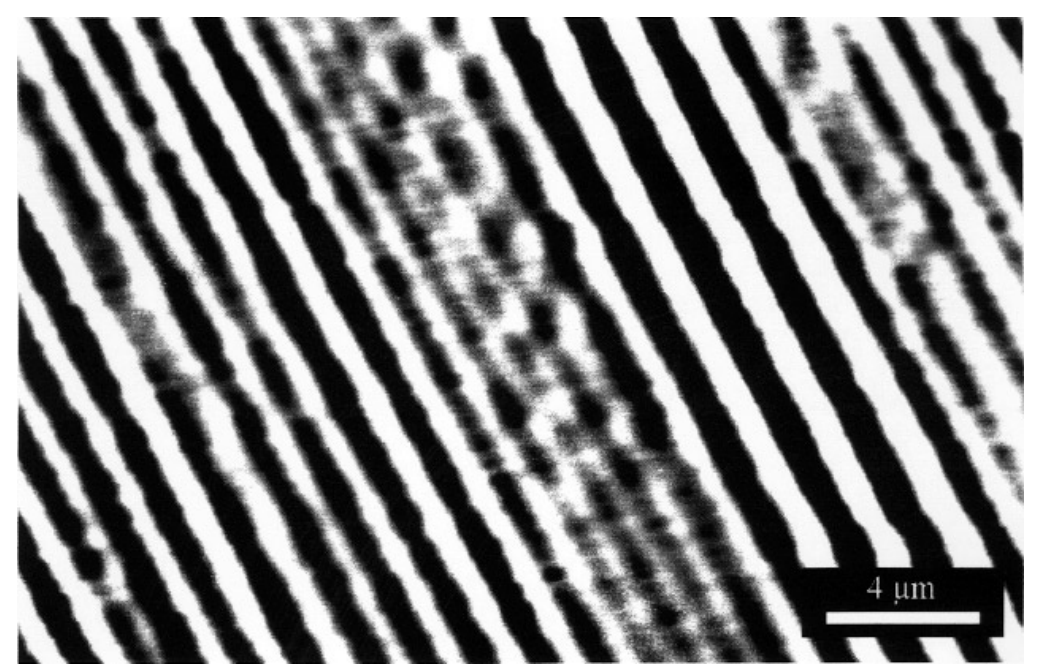

Figure 3. SEM-REBIC image of the $\mathrm{Cd}_{0.56} \mathrm{Hg}_{0.44} \mathrm{Te}$ cleaved surface shown at higher magnification in figure $1(a)$. 


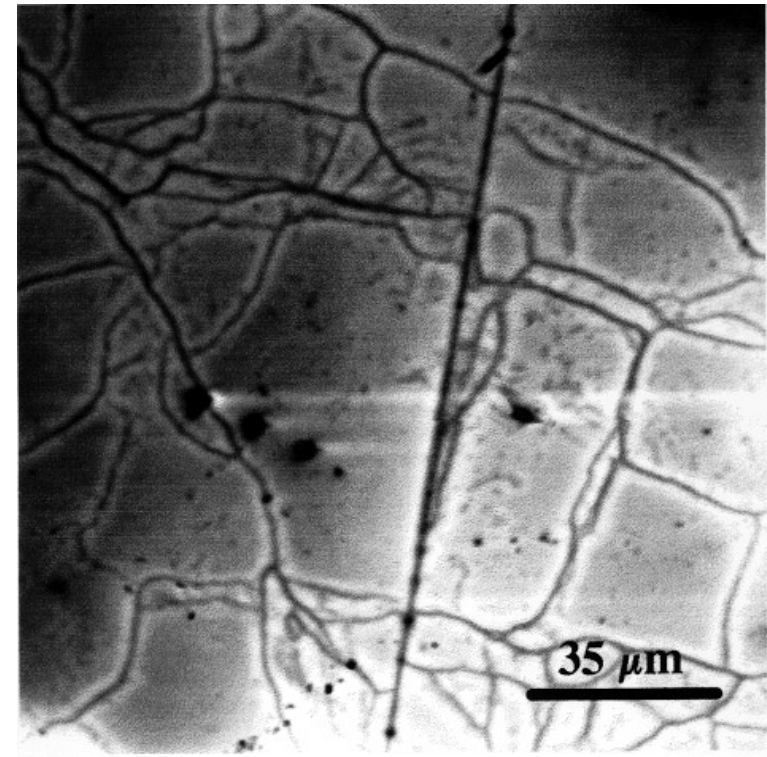

Figure 4. SEM-EBIC image of a CdTe crystal revealing different types of defects in the sample.

$I-V$ characteristics that were recorded for comparison. Measurements performed with different tips, on different samples and under different tunnelling conditions showed the reproducibility of our results, which lead us to conclude that the curves were representative of the material although
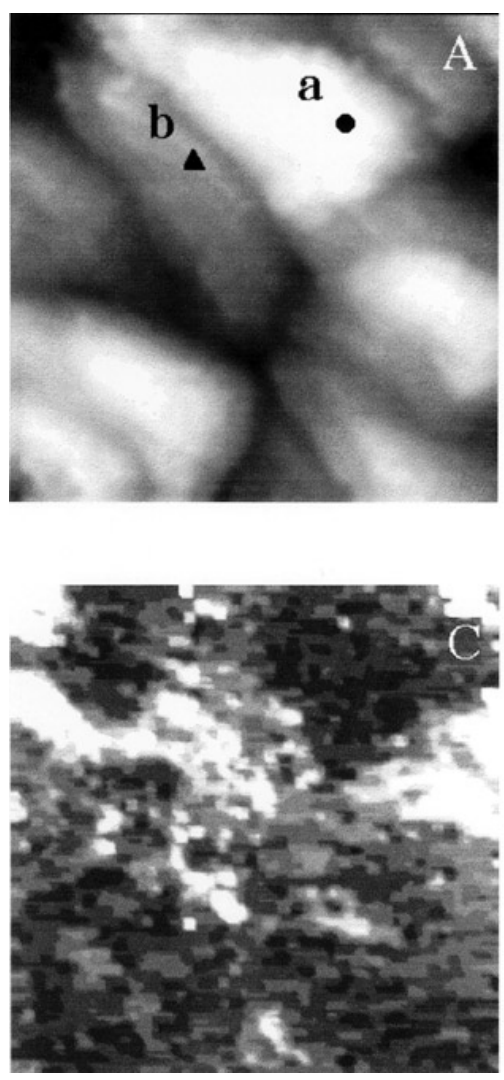

they were recorded in HV. It should also be mentioned that $I-V$ characteristics showing broad band gaps, which would be related to native oxides [23], were never observed in our investigations. These observations agree with previous reports on the extreme resistance of $\mathrm{Cd}_{x} \mathrm{Hg}_{1-x} \mathrm{Te}$ to oxidation [24]. Figure 6 shows the $(\mathrm{d} I / \mathrm{d} V) /(I / V)$ curves of two such regions of a p-type $\mathrm{Cd}_{0.56} \mathrm{Hg}_{0.44} \mathrm{Te}$ sample. In figure 6 , curve $a$, the width of the low conductance region on the voltage axis can be roughly estimated to be about $0.6 \mathrm{eV}$ which compares with the sample band gap of $0.64 \mathrm{eV}$. The asymmetry of the curve relative to the bias voltage agrees with the p-type conduction. Figure 6, curve $b$, shows a normalized differential conductance curve corresponding to a different area in which a narrower band gap is observed. Spatial variations of the energy gap detected from STM conductance curves have been reported previously for different materials, e.g. [25, 26]. In our case this observation agrees with the EBIC results revealing inhomogeneities of the electronic properties of the samples, which would be a consequence of local variations of surface stoichiometry. In $\mathrm{Cd}_{x} \mathrm{Hg}_{1-x} \mathrm{Te}$, defects can move from the bulk to the surface and in the opposite direction over hundreds of $\AA$, even at room temperature. This is related to the weakness of $\mathrm{Hg}$ bonding in the lattice and the high mobility of the $\mathrm{Hg}$ atoms, which migrate from or towards the surface creating positively charged interstitials or negatively charged vacancies [27]. No significant variations of the band gap width were observed
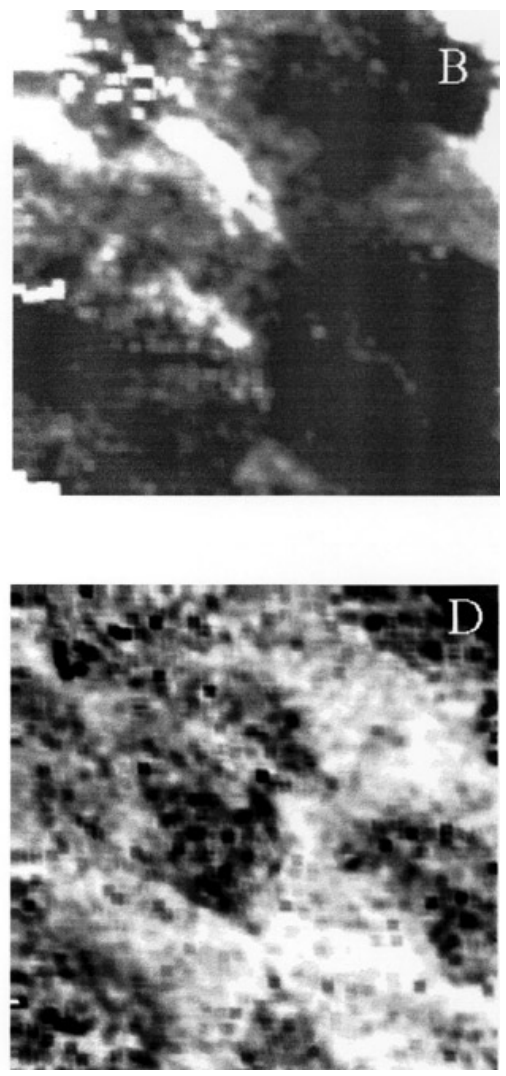

Figure 5. STM images of p-type $\mathrm{Cd}_{0.56} \mathrm{Hg}_{0.44} \mathrm{Te}$. (A) Topography image acquired at $-0.9 \mathrm{~V}$ sample bias, $0.2 \mathrm{nA}$ tunnelling current, $200 \times 200 \mathrm{~nm}^{2}$ in size. $(B)-(D)$ Corresponding ClTS images obtained at $(B)+1.15 \mathrm{~V},(C)+0.38 \mathrm{~V}$ and $(D)-0.38 \mathrm{~V}$ sample bias voltages. 


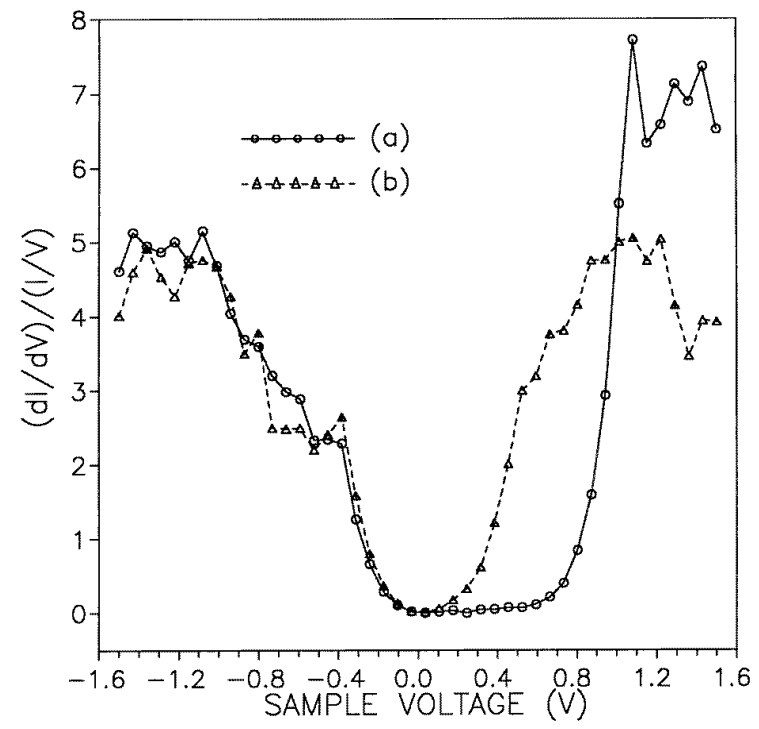

Figure 6. $(\mathrm{d} / / \mathrm{d} V) /(I / V)$ as a function of applied bias voltage curves recorded at the regions labelled ' $a$ ' and ' $b$ ' in figure $5(A)$.

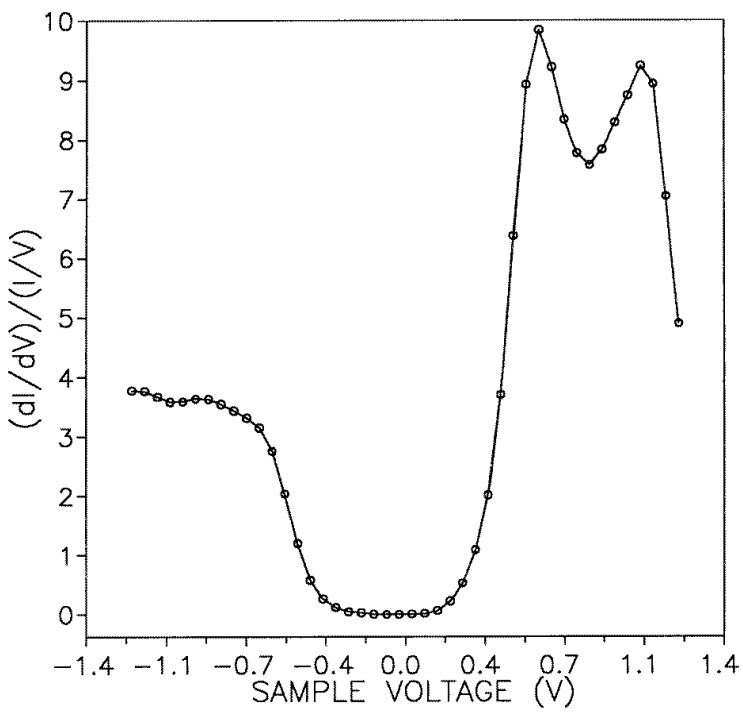

Figure 7. $(\mathrm{d} I / \mathrm{d} V) /(I / V)$ versus voltage curve obtained from an n-type $\mathrm{Cd}_{0.42} \mathrm{Hg}_{0.58} \mathrm{Te}$ sample.

in conductance curves recorded in regions different from the areas containing charged defects imaged by SEM-(R)EBIC micrographs.

A typical example of the $(\mathrm{d} I / \mathrm{d} V) /(I / V)$ dependence observed in the n-type $\mathrm{Cd}_{0.42} \mathrm{Hg}_{0.58} \mathrm{Te}$ samples is presented in figure 7. A surface band gap of about $0.4 \mathrm{eV}$ can be estimated from this curve, which is very close to the $0.45 \mathrm{eV}$ bulk band gap of these samples. Comparison of figures 6 and 7 clearly shows that the curves of n- and p-type samples appear shifted in opposite directions relative to the zero bias voltage, according to the conductivity type.

Figure 8 shows representative $(\mathrm{d} I / \mathrm{d} V) /(I / V)$ curves recorded at different points of a CdTe sample. As in the $\mathrm{Cd}_{x} \mathrm{Hg}_{1-x} \mathrm{Te}$ case, the shapes of the curves depend on the position where they were recorded on the sample. In

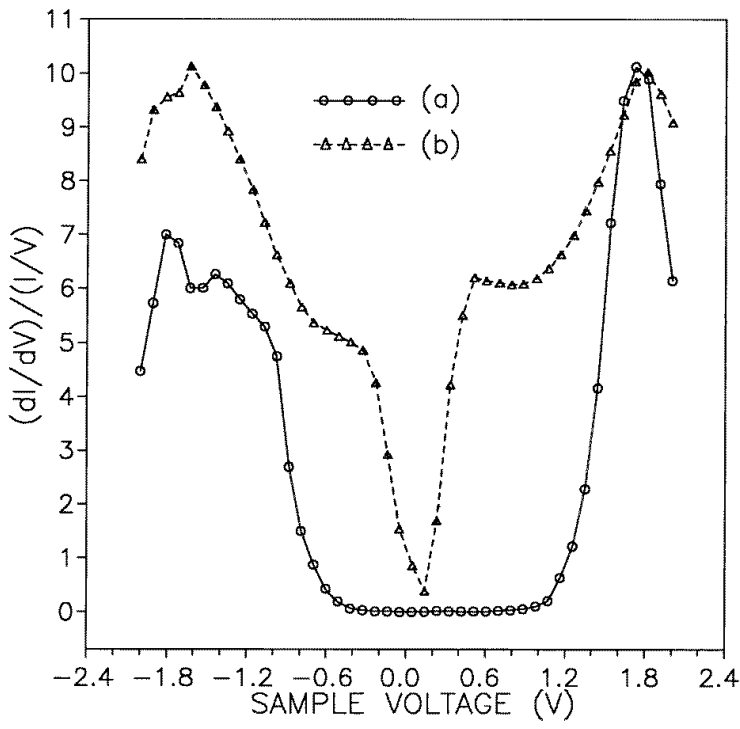

Figure 8. $(\mathrm{d} / / \mathrm{d} V) /(I / V)$ dependences on applied bias voltage recorded at two different positions on a CdTe sample.

particular, curve a shows an energy gap of about $1.4 \mathrm{eV}$ in agreement with the $1.5 \mathrm{eV}$ band gap of $\mathrm{CdTe}$, while curve $\mathrm{b}$ derives from an $I-V$ curve with a marked metallic character. This shows that the high inhomogeneity revealed in the EBIC images is also detected in the conductance curves with strong variations in surface band gap which would be related to nanoscaled clustering or precipitation processes.

\section{Conclusions}

STM-CITS enables the study of local electronic properties with $\mathrm{nm}$ resolution in undoped $\mathrm{CdTe}$, with low carrier concentration, and $\mathrm{Cd}_{x} \mathrm{Hg}_{1-x} \mathrm{Te}$ crystals. The use of a combined SEM-STM instrument allowed correlative studies of topography and electronic behaviour in both materials. In particular, regions with different recombination properties, observed in SEM-EBIC and REBIC images, show local variations of the band gap in the normalized differential conductance curves obtained by tunnelling spectroscopy.

\section{Acknowledgments}

This work was supported by DGICYT (Project PB931256) and by CICYT (Project IN93-0012). The help of Professor A M Baró, Dr A Asenjo and Dr J Gómez-Herrero throughout this work is gratefully acknowledged. G N Panin thanks Spanish MEC for a research grant.

\section{References}

[1] Panin G N and Yakimov E 1992 Semicond. Sci. Technol. A 7150

[2] Pal U, Fernández P, Piqueras J, Sochinskii N V and Dieguez E 1995 J. Appl. Phys. 781992 
[3] Panin G N, Fernández P and Piqueras J 1996 Semicond. Sci. Technol. 111354

[4] Castaldini A, Cavallini A, Fraboni B, Polenta L, Fernández P and Piqueras J 1996 Phys. Rev. B 547622

[5] Seehofer L, Falkenberg G, Johnson R L, Etgens V H, Tatarenko S, Brun D and Daudin B 1995 Appl. Phys. Lett. 671680

[6] Seehofer L, Etgens V H, Falkenberg G, Veron M B, Brun D, Daudin B, Tatarenko S and Johnson R L 1996 Surf. Sci. 347 L55

[7] Castro-Rodríguez R, Zapata-Torres M, Zapata-Navarro A, Oliva A I and Peña J L 1996 J. Appl. Phys. 79184

[8] Feenstra R M 1994 Surf. Sci. 299-300 965

[9] Hamers R J, Tromp R M and Demuth J E 1986 Phys. Rev. Lett. 561972

[10] First P N, Stroscio J A, Dragoset R A, Pierce P T and Celotta R J 1989 Phys. Rev. Lett. 631416

[11] Asenjo A, Gómez-Rodríguez J M and Baró A M 1992 Ultramicroscopy 42-44933

[12] Tanimoto M and Nakano Y 1990 J. Vac. Sci. Technol. A 8553

[13] Asenjo A, Buendía A, Gómez-Rodríguez J M and Baró A M 1994 J. Vac. Sci. Technol. B 121658

[14] Holt D B, Raza B and Wojcik A 1996 Mater. Sci. Eng. B 4214

[15] Stroscio J A, Feenstra R M and Fein A P 1986 Phys. Rev. Lett. 572579
[16] Lang N D 1986 Phys. Rev. B 345947

[17] Feenstra R M, Stroscio J A and Fein A P 1987 Surf. Sci. 181295

[18] Wasenczuk A, Willoughby A F W, Mackett P, O'Keefe E, Capper P and Maxey C D 1996 J. Cryst. Growth 159 1090

[19] Darchuk S D, Panin G N, Plyatsko S V, Sizov F F and Yakimov E B 1990 J. Phys. Chem. Solids 511333

[20] Panin G N and Yakimov E B 1991 J. Phys. IV, Colloq. C 6 C6-181

[21] Panin G N and Yakimov E B 1991 Microscopy of Semiconducting Materials 1991 (Inst. Phys. Conf. Ser. 117) ed A G Cullis and A R Long (Bristol: Institute of Physics) p 763

[22] Blumtritt H, Panin G N, Yakimov E B and Heydenrich J 1988 Phys. Status Solidi A 109 K3

[23] Wager J F and Rhiger D R 1985 J. Vac. Sci. Technol. A 3 212

[24] Spicer W E, Silberman J A, Lindau I, Sher A and Wilson J A 1983 J. Vac. Sci. Technol. A 11735

[25] Stroscio J A and Feenstra R M 1993 Methods Exp. Phys. 2795

[26] Edwards H, Derro D J, Barr A L, Markert T J and de Lozanne A L 1996 J. Vac. Sci. Technol. B 141217

[27] Nemirovsky Y and Bahir G H 1989 J. Vac. Sci. Technol. A 7206 